1 Universidade de Pernambuco (UPE) Nazaré da Mata (PE), Brasil.

djalma.guimaraes@upe.br

2 Universidade do Estado do Mato Grosso (Unemat) - Barra do Bugres (MT),

Brasil.

eduardo.oenning@unemat.br

3 Universidade Federal de Pernambuco (UFPE) Recife (PE), Brasil. medeirosdd@gmail.com

4 Universidade Federal de Pernambuco (UFPE) Recife (PE), Brasil. gileno.ferraz@gmail.com

\section{Mapeamento do perfil de demanda inadequada nos serviços de saúde pública no Brasil}

Mapping the profile of inappropriate demand in public health services in Brazil

Djalma Silva Guimarães Júnior' ${ }^{\mathbf{1}}$ Eduardo José Oenning Soares², Denise Dumke de Medeiros³, Gileno Ferraz Júnior4

RESUMO Este artigo investiga o perfil de uso inadequado em serviços de saúde pública no Brasil, através da análise das características do usuário e das circunstâncias de atendimento de usuários de call centers. São utilizadas informações de centrais de aconselhamento médico por telefone. Um modelo de regressão logística binária é proposto para estimar as relações entre o perfil do usuário, as circunstâncias de atendimento e a demanda inadequada. Impactaram significativamente para a probabilidade de demanda inadequada a maior complexidade da intenção inicial do usuário e o uso do sistema de serviço de saúde nas horas finais do dia.

PALAVRAS-CHAVE Administração de serviços de saúde. Telemedicina. Necessidades e demanda de serviços de saúde. Modelos logísticos.

ABSTRACT This article investigates the profile of inappropriate use in public health services in Brazil, through the analysis of user's characteristics and customer service circumstances of call centers. Information from telephone medical advice centers is used. A binary logistic regression model is proposed to estimate the relationships between the user profile, the service circumstances and the inadequate demand. The greater complexity of the user's initial intention and the use of the health service system in the final hours of the day were significantly impacted by the probability of inadequate demand.

KEYWORDS Health services administration. Telemedicine. Health services needs and demand. Logistic models. 


\section{Introdução}

Ao mesmo tempo em que diversos segmentos do setor público brasileiro enfrentam pressões para a minimização de custos devido as políticas de austeridade econômica, o setor de saúde pública, além de conviver com a escassez de recursos, ainda se defronta com a necessidade de elevação dos padrões de qualidade percebida pelos usuários. Um recurso de uso crescente em sistemas de saúde, ao redor do mundo, é a adoção de Tecnologias de Informação e Comunicação (TICs), que permitem aos provedores de serviços de saúde fornecer cuidados de saúde em tempo real para os usuários, disseminar informações que auxiliam na medicina preventiva e fornecer um serviço mais customizado, através de monitoramento e aconselhamento, ao mesmo tempo em que tendem a reduzir os custos, a partir da eliminação de procedimentos desnecessários e melhores práticas preventivas.

Uma parcela significativa dos gastos de sistemas de saúde é destinada a procedimentos considerados desnecessários. A intenção inicial do usuário, ao procurar um serviço médico, pode divergir da ação mais recomendada, fato que onera os custos do sistema (BOGDAN ET AL., 2004; BUNIK ET AL., 2007; CARRET; FASSA; DOMINGUES, 2009; GUIMARÃES JUNIOR ET AL., 2015; MCHALE ET AL., 2013). Um dos fatores que ocasionam o erro de julgamento dos usuários é a falta de informação adequada (CAMPBELL, 2000; MUKHERJEE; MCGINNIS, 2007).

Nesse contexto, emerge uma importante contribuição das TICs para o setor de saúde, um grupo de ferramentas denominado mobile health ( $m$-health), que consiste em qualquer instrumento eletrônico, tecnologia ou aplicativo projetado para a interação entre profissionais de saúde e usuários (HANDEL, 2011). $\mathrm{O}$ uso das ferramentas de $m$-health e, mais especificamente, de call centers de aconselhamento médico, pode fornecer informações aos usuários, auxiliando-os na tomada de decisão mais adequada ao demandarem serviços de saúde, ou seja, favorece uma demanda mais próxima da real necessidade do usuário.

$\mathrm{O}$ escopo de estudos do $m$-health tem-se expandido nos últimos anos. Guimarães Junior et al. (2015) apresentam o impacto de centrais de aconselhamento médico por telefone na redução de custos do sistema; Handel (2011) apresenta as principais aplicações de m-health em smartphones e tablets, através do desenvolvimento de inúmeros aplicativos; outro grupo de autores tem se preocupado com a qualidade percebida pelos usuários e a eficiência dos serviços (MECHAEL, 2009; AKTER ET AL., 2010).

A Constituição Federal Brasileira de 1988 foi um marco para a configuração do sistema de serviços de saúde no País, pois definiu a saúde como direito de todos e dever do Estado. Até a década de 1980, a prestação de serviços de saúde pública no País era de responsabilidade do Instituto Nacional de Assistência Médica e Previdência Social (Inamps), que foi gradualmente incorporado, no início da década de 1990, ao Ministério da Saúde (MS), dando origem ao Sistema Único de Saúde (SUS), que garante acesso universal, a todos os brasileiros, à assistência em saúde (BRASIL, 2006).

Paralelamente, a partir da década de 1990, o País passou por um processo de afastamento da ação estatal da economia. No setor de saúde, isso contribuiu para uma maior participação de entidades privadas no sistema de serviços de saúde, com sistemas de saúde suplementar (SESTELO; SOUZA; BAHIA, 2013). Ou seja, de um lado, ocorreu a universalização do direito à saúde, e do outro, uma alternativa de saúde suplementar. Mas tal configuração do sistema de serviços de saúde brasileiro não tem garantido a satisfação com os serviços prestados. Segundo o Conselho Federal de Medicina (2014), 93\% dos brasileiros consideram o sistema de serviços de saúde público e privado, no Brasil, ruim ou muito ruim. Outro indicador do desempenho do atendimento de saúde no País, o Índice do Desempenho do Sistema Único de Saúde (IDSUS), apontou 
que apenas 13,57\% dos municípios brasileiros possuem índice superior a 6 , em uma escala que varia entre 0 e 10 (BRASIL, 2015).

No atual ambiente de recessão econômica e diante da já mencionada insatisfação com os sistemas de saúde, algumas proposições conservadoras têm surgido na direção de onerar ainda mais a população brasileira, a exemplo de propostas de copagamento para complemento ao financiamento do SUS (HOLST; GIOVANELLA; ANDRADE, 2016).

Nesse sentido, este artigo objetiva apresentar uma análise sobre o perfil de uso inadequado em serviços de saúde pública, no Brasil, através das características do usuário e das circunstâncias de seu atendimento. Ao final, são propostas práticas que apoiem ações preventivas e, ao mesmo tempo, minimizem o uso inadequado. Isto é possível através de um modelo de regressão logística que estime quais são os fatores determinantes para tal demanda, através de dados coletados por uma central de aconselhamento médico por telefone. O resultado desta pesquisa pode auxiliar o gestor de serviços de saúde no direcionamento de estratégias promocionais para usuários com maior probabilidade de demanda incorreta, bem como no aprimoramento de serviços de triagem e classificação de usuários, e desenvolvimento de ferramentas de $m$-health adaptadas às necessidades dos usuários do SUS.

\section{Métodos}

Os dados utilizados foram cedidos por uma empresa prestadora de serviços de aconselhamento médico, através de centrais de call centers, situada na cidade de Recife (PE), que atende usuários do SUS de vários municípios do Brasil. A amostra de dados compreende todas as ligações recebidas pela central de aconselhamento médico durante 18 dias seguidos, no ano de 2012, totalizando 380 observações.

O usuário do SUS, ao ligar para uma central de atendimento, possui uma intenção inicial de uso do serviço médico, que é confrontada com a recomendação proposta por médicos. Os casos que apresentam convergência entre a demanda do usuário e a recomendação médica são considerados casos de demanda adequada. Já as situações nas quais ocorre divergência, tem-se uma demanda inadequada. Neste contexto de telemedicina, é possível observar algumas características do usuário (idade e sexo) e as circunstâncias do atendimento (dia da semana, hora e intenção inicial do usuário).

As características do perfil de demanda inadequada do sistema público de saúde foram testadas através de um modelo de regressão logística binária, que possuía como variável dependente a intenção do usuário: demanda correta ao ligar para a central (0) e demanda incorreta ao ligar para a central (1). As variáveis independentes foram: sexo masculino (1) e feminino (0); idade (em anos); intenção inicial: não fazer nada (0), marcar consulta (1) e ir a uma urgência/emergência (2); horário da ligação: 00:01-06:00 (1), 06:01-12:00 (2), 12:01-18:00 (3) e 18:01-00:00 (4); e dia da semana: segunda (1), terça (2), quarta (3), quinta (4), sexta (5), sábado (6) e domingo (7). Todas as análises estatísticas foram feitas com o software XLSTAT (ADDINSOFT, 2016).

No modelo de regressão foi utilizado o método Stepwise Forward, que insere, a cada iteração, as variáveis significativas do modelo, restando, no final, apenas os parâmetros que possuem um relacionamento consistente com a variável dependente.

A regressão logística foi utilizada para determinar qual é o perfil ou quais são as circunstâncias que induzem à demanda inadequada de serviços de saúde, de forma a fornecer informações úteis para gestores, com vistas à minimização de situações geradoras de deseconomias para o sistema. Tal técnica de análise multivariada de dados foi utilizada em exitosos casos, a fim de determinar as variáveis mais significativas para a probabilidade de ocorrência de um evento. 
Weltermann et al. (2013) utilizaram a regressão logística para determinar o conhecimento dos entrevistados sobre os sintomas do Acidente Vascular Cerebral (AVC), a partir de informações prévias destes sobre AVC e dados sociodemográficos.

Os autores informam que não existiu conflito de interesses na elaboração da pesquisa, bem como não houve nenhum tipo de suporte financeiro. $\mathrm{O}$ estudo foi submetido à apreciação do Comitê de Ética e Pesquisa da Universidade do Estado de Mato Grosso (Unemat), tendo declaração favorável à utilização dos dados no parecer $n^{0}$ 008/2014/ REC/Unemat.

\section{Resultados e discussão}

A tabela 1 apresenta algumas informações de perfil demográfico e características do atendimento, distribuídas entre demanda adequada e inadequada. Em relação ao dia da semana, existe um maior número de ocorrências de demanda inadequada na segunda-feira. O horário mais frequente de demanda inadequada se encontra na faixa compreendida entre $13 \mathrm{~h}$ e $18 \mathrm{~h}$. Predomina a demanda inadequada entre indivíduos do sexo masculino, na faixa etária de 0-15 anos e com intenção inicial de ir à emergência.

Tabela 1. Perfil da amostra e distribuição de demanda adequada e inadequada

\begin{tabular}{|c|c|c|c|}
\hline Variáveis & & $\begin{array}{c}\text { Intenção adequada } 117 \\
(30,8 \%)\end{array}$ & $\begin{array}{c}\text { Intenção inadequada } 263 \\
(69,2 \%)\end{array}$ \\
\hline \multirow{7}{*}{$\begin{array}{l}\text { Dia da semana } \\
\chi^{2}=4,817 \\
P=0,568\end{array}$} & Segunda & $14(12 \%)$ & $44(16,7 \%)$ \\
\hline & Terça & $14(12 \%)$ & $33(12,5 \%)$ \\
\hline & Quarta & $16(13,7 \%)$ & $41(15,6 \%)$ \\
\hline & Quinta & $22(18,8 \%)$ & $38(14,4 \%)$ \\
\hline & Sexta & $18(15,4 \%)$ & $41(15,6 \%)$ \\
\hline & Sábado & $12(10,3 \%)$ & $34(12,9 \%)$ \\
\hline & Domingo & $21(17,9 \%)$ & $32(12,2 \%)$ \\
\hline \multirow{4}{*}{$\begin{array}{l}\text { Hora } \\
\chi^{2}=4,357 \\
P=0,225\end{array}$} & 01:00-06:00 & $9(7,7 \%)$ & $13(4,9 \%)$ \\
\hline & 07:00-12:00 & $36(30,8 \%)$ & $61(23,2 \%)$ \\
\hline & 13:00-18:00 & $39(33,3 \%)$ & $96(36,5 \%)$ \\
\hline & 19:00-00:00 & $33(28,2 \%)$ & $93(35,4 \%)$ \\
\hline \multirow{2}{*}{$\begin{array}{l}\text { Sexo } \\
\chi^{2}=0,335 \\
P=0,563\end{array}$} & Masculino & $188(71,5 \%)$ & $87(74,4 \%)$ \\
\hline & Feminino & $75(28,5 \%)$ & $30(25,6 \%)$ \\
\hline \multirow{6}{*}{$\begin{array}{l}\text { Idade } \\
\chi^{2}=2,632 \\
P=0,757\end{array}$} & $0-15$ & $39(33,3 \%)$ & $89(33,8 \%)$ \\
\hline & $16-24$ & $32(27,4 \%)$ & $65(24,7 \%)$ \\
\hline & $25-34$ & $28(23,9 \%)$ & $69(26,2 \%)$ \\
\hline & $35-44$ & $8(6,8 \%)$ & $24(9,1 \%)$ \\
\hline & $45-59$ & $5(4,3 \%)$ & $11(4,3 \%)$ \\
\hline & 60 ou mais & $5(4,3 \%)$ & $5(1,9 \%)$ \\
\hline \multirow{3}{*}{$\begin{array}{l}\text { Intenção inicial } \\
\chi^{2}=26,314 \\
P=0\end{array}$} & Não fazer nada & $24(20,5 \%)$ & $11(4,2 \%)$ \\
\hline & Marcar consulta & $33(28,2 \%)$ & $79(30,0 \%)$ \\
\hline & Ir à urgência/emergência & $173(65,8 \%)$ & $60(51,3 \%)$ \\
\hline
\end{tabular}


Como observado na tabela 1 , cerca de $69,2 \%$ das intenções de uso do serviço de saúde público foram consideradas inadequadas. Ou seja, a maioria dos usuários possuía uma intenção errada ao demandar o serviço de saúde pública, no momento da ligação.

O resultado do modelo de regressão proposto pode ser observado na tabela 2 .

\begin{tabular}{lrrrrr}
\hline \multicolumn{1}{l}{ Tabela 2. Modelo de regressão } & & & & \\
\hline & B & Sig $^{\star}$ & OR & 95\% IC for OR & \\
\cline { 5 - 6 } & & & & Inferior & Superior \\
\hline Intenção inicial &, 683 &, 000 & 1,979 & 1,425 & 2,750 \\
Hora &, 315 &, 013 & 1,371 & 1,069 & 1,758 \\
Constant & $-1,836$ &, 002 &, 159 & & \\
\hline
\end{tabular}

*Nível de significância.

A regressão mostra que apenas duas variáveis são consideradas significativas para a demanda inadequada, segundo o modelo proposto, a intenção inicial do usuário e a hora pretendida para o atendimento. As demais variáveis (dia da semana, sexo e idade) foram consideradas não significativas para a modelagem. Em relação à intenção inicial, cada incremento unitário na complexidade do atendimento pretendido eleva em $97,9 \%$ as chances de o usuário apresentar demanda inadequada, Odds Ratio (OR) de 1,979, tendo Intervalo de Confiança (IC) a 95\% de 1,425-2,750. Em relação à hora, à medida que aumentam as classes de horas consideradas, a chance de o usuário apresentar intenção inadequada se eleva em 37,1\% (OR: 1.371; 95\% IC: 1.069-1.758). O modelo proposto se mostra estatisticamente significante, pois rejeita a hipótese nula $(\beta 0$ e $\beta 1=0)$ no teste de razão de verossimilhança. Logo, os parâmetros estimados são significativos, fato endossado pelo baixo p-valor.

Em um contexto no qual o sistema de serviços de saúde enfrenta pressões por redução de custos e melhoria na qualidade, é vital para as organizações públicas a adoção de estratégias e procedimentos que vão de encontro a tais demandas. O uso de TICs corrobora tais objetivos, através da racionalização do uso do sistema e da disponibilização de mais informações para os usuários, que auxiliem na prevenção e no tratamento de doenças, bem como na minimização da alocação de recursos que seriam empregados de forma desnecessária em demandas inadequadas.

A variável intenção inicial confirma os trabalhos de Bunik et al. (2007) e Guimarães Junior et al. (2015), nos quais o usuário desassistido em serviços de aconselhamento possui maior propensão ao uso inadequado. Quanto maior a complexidade da intenção inicial do usuário, maior a chance de uso inadequado do sistema público de saúde. Dado o percentual de inadequação de demanda na amostra, percebe-se um grande potencial de agregação de eficiência ao sistema através da minimização do uso inadequado. Logo, conforme os resultados deste estudo e da literatura, uma fonte de economia de recursos no sistema de serviços de saúde pública brasileiro pode se derivar de políticas de aconselhamento médico, que direcionem os usuários de acordo com sua real necessidade, diminuindo o fluxo para os centros de tratamento mais intensivo, minimizando custos e contribuindo para a melhoria do nível de serviço e qualidade percebida. 
Em relação à hora, à medida que as horas avançam durante o dia, ocorre um crescimento na probabilidade de demanda inadequada, e tal característica corrobora os resultados de outros autores, como os de Carret, Fassa e Domingues (2009), McHale et al. (2013) e Guimarães Junior et al. (2015). Assim, os gestores de serviços públicos de saúde devem preocupar-se em fornecer serviços de aconselhamento e triagem de forma mais intensiva nos períodos da tarde e da noite, nos quais existe a maior possibilidade de uso inadequado.

Dessa forma, para o serviço público de saúde no Brasil, são de vital importância o acompanhamento e a criação de campanhas públicas para a conscientização e a informação dos cidadãos, pois, de acordo com a amostra de dados, $69,2 \%$ das demandas de saúde foram consideradas, por uma central de aconselhamento, como inadequadas. $\mathrm{O}$ percentual de intenção inadequada aponta para o potencial de economias de recursos no sistema de serviços de saúde, a partir do uso de call centers de aconselhamento médico, auxiliando na identificação e prevenção de intenções inadequadas.

\section{Conclusões}

Tendo em vista o crescente debate sobre estado mínimo e pressões por políticas conservadoras, como, por exemplo, a proposta de copagamento como complemento ao financiamento do SUS, é de suma importância que sejam levantadas novas possibilidades para a redução de custos e a melhoria da eficiência do sistema de serviços de saúde pública, para contrapor tais propostas e, assim, garantir um sistema de serviços de saúde de qualidade e gratuito.

Nesse sentido, este estudo proporcionou a identificação do perfil do usuário do sistema público de saúde e as circunstâncias de atendimento que impactam significativamente na demanda inadequada deste sistema em municípios brasileiros, através do uso de centrais de aconselhamento médico por telefone. Foi constatado que uma maior complexidade da intenção inicial do usuário e a necessidade de uso do sistema de serviços de saúde nas horas finais do dia tendem a apresentar maior probabilidade de uso inadequado.

Levando, ainda, em consideração que a grande maioria dos usuários do sistema de serviços de saúde procura tais serviços inadequadamente, justifica-se o uso de centrais de aconselhamento médico - seja com plataformas m-health -, bem como campanhas públicas para conscientizar e informar os cidadãos, o que oportunizará melhoria na eficiência do sistema de serviços de saúde pública, bem como redução de custos.

Apesar da limitação quanto ao público por ela investigado, acredita-se que o uso de uma central de aconselhamento médico via call center tenha se mostrado muito útil para coleta de dados, proporcionando bons resultados para as ilações do presente estudo. Contudo, recomenda-se, para futuras pesquisas, que sejam ampliados os dados pesquisados, sendo estes, por exemplo, diretamente obtidos através de prontuários médicos de hospitais inclusos no sistema de serviços de saúde pública, para reforçar ou refutar essas constatações.

\section{Colaboradores}

Djalma Silva Guimarães Junior e Eduardo José Oenning Soares contribuíram substancialmente para a concepção e o planejamento do estudo, realizaram a análise estatística e ajudaram a redigir o manuscrito. Gileno Ferraz e Denise Dumke de Medeiros conceberam o estudo e participaram do planejamento, da revisão crítica e da aquisição de dados. Todos os autores foram responsáveis pela reformulação e revisão do conteúdo intelectual deste artigo, bem como pela leitura e aprovação da versão final do manuscrito. 


\section{Referências}

ADDINSOFT. XLSAT Statistical software: version

2016.3. Paris, 2016.

AKTER. S.; D’AMBRA, J., RAY, P. Service quality of mHealth platforms: development and validation of a hierarchical model using PLS. Electronic Markets, v. 20, p. 209-227, 2010. Disponível em: <http://ro.uow.edu.au/ cgi/viewcontent.cgi? article $=3964 \&$ context $=$ commpape rs>. Acesso em: 5 fev. 2017.

BOGDAN, G. M. et al. Evaluating patient compliance with nurse advice line recommendations and the impact on healthcare costs. American Journal of Managed Care, Old Bridge, v. 10, n. 8, p. 534-542, ago. 2004. Disponível em: <https://www.ncbi.nlm.nih.gov/pubmed/15352529>. Acesso em: 5 fev. 2017.

BRASIL. Ministério da Saúde. Entendendo o SUS. Brasília, DF: Ministério da Saúde, 2006. Disponível em: <http://portalsaude.saude.gov.br/images/pdf/2013/ agosto/28/cartilha-entendendo-o-sus-2007.pdf>. Acesso em: 20 dez. 2015.

Ministério da Saúde. Índice de Desempenho do Sistema Único de Saúde. Brasília, DF: Ministério da Saúde, 2015. Disponível em: <http://idsus.saude.gov.br/ documentos/Apresentacao_IDSUS.pdf> Acesso em: 20 fev. 2017.

BUNIK, M. et al. A Pediatric Telephone Call centers: How Do They Affect Health Care Use and Costs? Pediatrics, Springfield, v. 119, n. 2, p. 305-313, 2007. Disponível em: <https://www.ncbi.nlm.nih.gov/pubmed/17272593>. Acesso em: 20 fev. 2017.

CAMPBELL, R. J. Consumer health, patient education, and the internet. The Internet Journal of Health, [S. 1.], v. 2, n. 2, 2000. Disponível em: <http://ispub.com/ IJH/2/2/13424>. Acesso em: 20 fev. 2017.

CARRET, M. L.; FASSA, A. C.; DOMINGUES, M. R. Inappropriate use of emergency services: a systematic review of prevalence and associated factors. Caderno de Saúde Pública, Rio de Janeiro, v. 25, n. 1, p. 7-28, jan. 2009. Disponível em: <http://www.
scielo.br/scielo.php?script=sci_arttext\&pid=S0102-

-311X2009000100002>. Acesso em: 20 fev. 2017.

CONSELHO FEDERAL DE MEDICINA. Opinião dos brasileiros sobre o atendimento na área da saúde. 2014 Disponível em: <http://portal.cfm.org.br/images/PDF/ apresentao-integra-datafolha203.pdf>. Acesso em: 20 dez. 2014.

GUIMARÃES JUNIOR, D. S. et al. Attributes and circumstances that induce inappropriate health services demand: a study of the health sector in Brazil, BMC Health Services Research, Londres, v. 15, n. 65, 2015. Disponível em: <https://www.ncbi.nlm.nih.gov/pmc/ articles/PMC4339295/>. Acesso em: 20 fev. 2017. Não paginado.

HANDEL, M. J. Mhealth (mobile health): using apps for healthand wellness. Explore, Nova Iorque, v. 7, n. 4, p. 256-261, jul./ago. 2011. Disponível em: <https://www. ncbi.nlm.nih.gov/pubmed/21724160>. Acesso em: 20 fev. 2017.

HOLST, J.; GIOVANELLA, L.; ANDRADE, G. C. L. Porque não instituir copagamento no Sistema Único de Saúde: efeitos nocivos para o acesso a serviços e a saúde dos cidadãos. Saúde em Debate, Rio de Janeiro, v. 40, n. esp., p. 213-226, dez. 2016. Disponível em: <http:// www.scielo.br/pdf/sdeb/v40nspe/0103-1104-sdeb-40spe-0213.pdf>. Acesso em: 20 fev. 2017.

MCHALE, P. et al. Who uses emergency departments inappropriately and when - a national cross-sectional study using a monitoring data system. BMC Medicine, Londres, v. 13, n. 11, dez. 2013. Disponível em: < https:// www.ncbi.nlm.nih.gov/pubmed/24330758>. Acesso em: 20 fev. 2017.

MECHAEL, P. The case for mHealth in developing countries. Innovations: Technology, Governance, Globalization, Cambridge, v. 4, n. 1, p. 103-118, abr. 2009. Disponível em: <http://www.mitpressjournals. org/doi/10.1162/itgg.2009.4.1.103>. Acesso em: 20 fev. 2017. 
MUKHERJEE, A.; MCGINNIS, J. E-healthcare: an analysis of key themes in research, International Journal of Pharmaceutical and Healthcare Marketing, Bingley, v. 1, n. 4, p. 349-363, 2007. Disponível em: <http://www.iupui.edu/ j21099/courses/readings/ mukherjee.pdf>. Acesso em: 20 fev. 2017

SESTELO, J. A. F.; SOUZA, L. E. P. F.; BAHIA, L.

Private health insurance in Brazil: Approaches to public/private patterns in healthcare. Caderno de Saúde Pública, Rio de Janeiro, v. 29, n. 5, p. 851-866, maio 2013. Disponível em: <http://www.scielo.br/pdf/csp/ v29n5/04.pdf>. Acesso em: 20 fev. 2017.
WELTERMANN, B. M. et al. Stroke knowledge among diabetics: a cross-sectional study on the influence of age, gender, education, and migration status. $B M C$ Neurology, Londres, v. 13, n. 202, dez. 2013. Disponível em: <https://www.ncbi.nlm.nih.gov/pmc/articles/ PMC3867625/>. Acesso em: 20 fev. 2017. Não paginado.

Recebido para publicação em março de 2017

Versão final em julho de 2017

Conflito de interesses: inexistente

Suporte financeiro: não houve 